\title{
2 Stigmatisierung: Implikationen für die Kommunikation mit adipösen Patienten
}

\author{
Anja Hilbert und Miriam Geiser
}

Im Bus, im Flugzeug, beim Kleiderkauf und in vielen anderen Situationen erleben adipöse Menschen ein Umfeld, in das sie nicht hineinpassen. Auch in vielen zwischenmenschlichen Interaktionen, sei es in der Schule, am Arbeitsplatz oder auf der Straße berichten Übergewichtige von Diskriminierung aufgrund ihres Gewichts. Selbst vor Klinik oder Arztpraxis machen negative Einstellungen gegenüber adipösen Patienten keinen Halt. Ob es zu enge Stühle, ein kritischer Blick des Pflegepersonals, eine abwertende Bemerkung des Zimmernachbarn oder der gut gemeinte ärztliche Ratschlag „Nehmen Sie einfach ein bisschen $a b$ “ ist, viele Übergewichtige fühlen sich in Einrichtungen des Gesundheitswesens nicht wohl, schämen sich für ihr Gewicht oder vermeiden es gänzlich, zum Arzt zu gehen. Dieses Kapitel vermittelt einen Einblick in die Stigmatisierung von Adipositas in verschiedenen Lebensbereichen, hauptsächlich aber im medizinischen Setting, und leitet Empfehlungen für einen nicht-stigmatisierenden, gesundheitsförderlichen Umgang mit adipösen Patienten ab.

\subsection{Stigmatisierung und soziale Diskriminierung von adipösen Menschen}

Als soziales Stigma wird eine Eigenschaft bezeichnet, die einen Menschen von anderen unterscheidet und die eine Abwertung beinhaltet. Adipösen Menschen wird beispielsweise zugeschrieben, faul, willensschwach, gefräßig und unattraktiv zu sein (Puhl u. Latner 2007; Brownell et al. 2005). Wenn es aufgrund dieses Adipositasstigmas zu einer tatsächli- chen Ungleichbehandlung von adipösen Menschen kommt, spricht man von gewichtsbezogener Diskriminierung.

Gewichtsbezogene Stigmatisierung und Diskriminierung erfahren adipöse Menschen in vielen verschiedenen Lebensbereichen, u.a. in persönlichen Beziehungen, in der Schule, am Arbeitsplatz und auch im Gesundheitswesen. Schon im Vorschulalter werden „dicke Kinder“ ausgeschlossen und gehänselt, im Jugend- und Erwachsenenalter gelten Übergewichtige als wenig attraktiv und haben Nachteile bei der Partnerwahl. Zusätzlich kommt es zu Benachteiligungen durch Lehrer und Arbeitgeber (Puhl u. Brownell 2001). Im Alltag stellen zu enge Sitze in Verkehrsmitteln oder öffentlichen Räumen Hindernisse dar. In den Medien werden adipöse Menschen häufig stereotyp dargestellt, und vielfach wird der Eindruck erweckt, dass Abnehmen kein Problem sei, wenn man nur wolle (Hilbert u. Ried 2009).

In vielen verschiedenen Lebensbereichen werden adipöse Menschen stigmatisiert und diskriminiert.

Wie Abbildung 1 zeigt, geht gewichtsbezogene Diskriminierung am häufigsten von Familienmitgliedern aus, z.B. in Form von kritischen Kommentaren über Figur und Gewicht. Gewichtsbezogene Diskriminierung in Familie oder Partnerschaft wird von den Betroffenen als besonders belastend erlebt. 


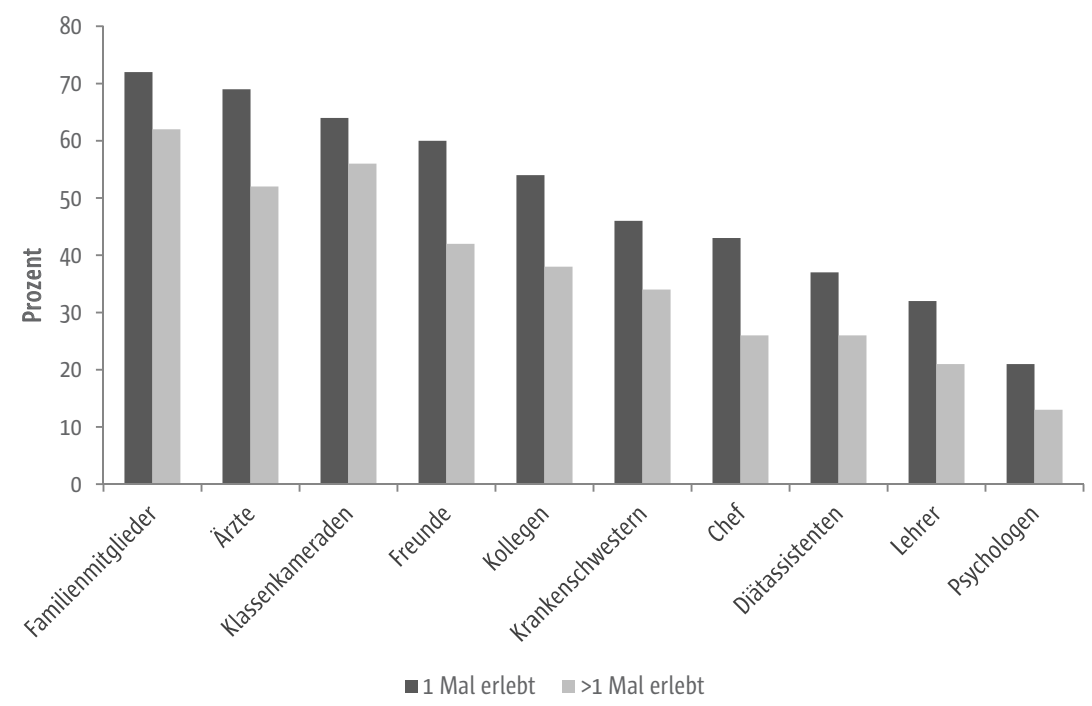

Abb. 1 Berichtete gewichtsbezogene Diskriminierung adipöser Frauen ( $N=2.449$ ) (Daten aus Puhl u. Brownell 2006)

Nicht zu unterschätzen ist auch, dass adipöse Menschen selbst eine negative Einstellung zum Übergewicht haben - sie werten sich daher selbst ab. Auch finden sie im Gegensatz zu anderen stigmatisierten Gruppen (z.B. Afro-Amerikanern) wenig Unterstützung unter ihresgleichen (Crandall 1994).

\section{Adipöse Menschen werten sich selbst aufgrund ihres Übergewichts ab und identifizieren sich nicht positiv mit der Gruppe der Übergewichtigen.}

Abbildung 1 verdeutlicht auch, dass gewichtsbezogene Stigmatisierung und Diskriminierung von adipösen Menschen im Gesundheitswesen weit verbreitet ist. Adipositas wird häufig als Verhaltensproblem angesehen, das durch zu wenig Bewegung und Überessen selbstverschuldet ist (Hilbert et al. 2008). Bereits Medizinstudenten empfinden abwertende Witze über adipöse Patienten nicht als unangebracht, weil sie den Patienten selbst die Schuld für ihr Übergewicht zuweisen (Wear et al. 2006). Selbst bei Diätassistenten und Ärzten, die auf Gewichtsreduktionsbehandlung spezialisiert sind, konnten stigmatisierendeEinstellungen festgestellt werden. So wird adipösen Patienten fehlende Willensstärke und Selbstkontrolle sowie mangelnde Hygiene zugeschrieben. Zudem gehen Behandler häufig davon aus, dass adipöse Patienten wenig „compliant“ sind und einen schlechteren Therapieerfolg als normalgewichtige Patienten ha- ben. Motivationsprobleme werden als Grund dafür angenommen, dass adipöse Patienten nicht abnehmen. Hingegen schätzen adipöse Patienten sich selbstals deutlich motivierter ein, als ihre behandelnden Ärzte es tun (Befort et al. 2006).

\section{Stigmatisierende Einstellungen sind im Gesundheits- wesen verbreitet und können Auswirkungen auf die Behandlung haben.}

Je schwerer ein Patient, als desto ungesünder und weniger diszipliniert wird er von Ärzten eingeschätzt und desto weniger haben Ärzte den Wunsch und die notwendige Geduld, dem Patienten zu helfen (Hebl u. Xu 2001). Auch Überforderung könnte eine Rolle spielen: In verschiedenen Untersuchungen gaben Ärzte an, dass sie für die Behandlung der Adipositas nicht hinreichend qualifiziert sind, nicht von einem Erfolg der Behandlung ausgehen und weniger Behandlungszeit mit übergewichtigen im Vergleich zu normalgewichtigen Patienten verbringen (Puhl $u$. Heuer 2009). Dies kann auch erklären, weshalb nur etwa 40\% der Allgemeinmediziner das Thema Gewichtsreduktion mit ihren adipösen Patienten überhaupt ansprechen (Hebl u. Xu 2001). Hingegen erleichtert ein umfangreicheres Wissen über Gewichtsreduktion Allgemeinmedizinern den Umgang mit diesem Thema und weckt weniger Frustration und Pessimismus (Ferrante et al. 2009). 
Lediglich 40\% der Ärzte sprechen mit adipösen Patienten über Gewichtsreduktion.

Nicht nur Ärzte berichten, dass sie weniger Zeit mit adipösen Patienten verbringen, sondern auch die $\mathrm{Pa}-$ tienten selbst geben an, weniger Behandlungszeit zu erhalten als normalgewichtige Patienten. Abbildung 1 zeigt, dass sich etwa 50\% der befragten adipösen Frauen mehrmals von Ärzten diskriminiert fühlten. Die erlebte Diskriminierung könnte auch ein Grund dafür sein, dass adipöse Patienten häufiger Arzttermine wieder absagen oder verschieben und mit der medizinischen Versorgung weniger zufrieden sind als normalgewichtige Patienten. Einerseits haben sie das Gefühl, dass sie immer wieder Diät verschrieben bekommen, ohne dass auf die Gründe für ihr Übergewicht eingegangen wird. Andererseits ist es vielen Patienten auch unangenehm, das Thema Übergewicht überhaupt anzusprechen, sodass sie es vermeiden, präventive Arztbesuche wahrzunehmen. So konnte zum Beispiel gezeigt werden, dass adipöse Frauen seltener als normalgewichtige Frauen an Screenings für Brust- und Gebärmutterhalskrebs teilnehmen (Ferrante et al. 2010).

Adipöse Patienten nennen die folgenden Gründe für die Vermeidung von Arztbesuchen:

- Scham über das eigene Gewicht,

- Unbehagen beim Ausziehen,

- Angst, gewogen zu werden,

- Unbehagen bei der Untersuchung und Berührung bestimmter Körperteile,

- ungebetene Ratschläge zu erhalten,

- Gefühl, nicht ernst genommen zu werden,

- Gefühl, dass das Gewicht als Begründung für jegliche Beschwerden herangezogen wird, sowie

- unzureichende Ausstattung (z.B. zu kleine Behandlungstische) (Drury u. Louis 2002; Hebl et al. 2003).

Welche psychischen Auswirkungen hat gewichtsbezogene Stigmatisierung insgesamt auf adipöse Menschen? Obwohl in der Allgemeinbevölkerung kein Zusammenhang zwischen Diskriminierungserfahrungen, depressiven Symptomen und einem negativen Selbstwert gefunden wurde, zeigen Patienten in klinischen Settings umso mehr psychische Auffälligkeiten, je mehr sie gewichtsbezogene Diskriminierung erlebt haben. Zudem wurden Diskriminierungserfahrungen mit Ängsten und einer stärkeren Unzufriedenheit mit dem eigenen Körper in Verbindung gebracht. Im Unterschied zum Erwachsenenalter sind gewichtsbezogene Hänseleien im Kindes- und Jugendalter ein Prädiktor für einen geringeren Selbstwert, mehr Unzufriedenheit mit dem eigenen Körper, Essanfälle und Diätverhalten, eine beeinträchtigte Lebensqualität und auch depressive Symptome bis hin zu Suizidgedanken nach mehreren Jahren (Hilbert 2008). Diese Ergebnisse weisen insgesamt darauf hin, dass gewichtsbezogene Diskriminierung psychisches Leid in vulnerablen Gruppen erhöht.

\subsection{Empfehlungen zur Kommunikation mit adipösen Patienten}

Um Schamgefühle und Unwohlsein in medizinischen Behandlungen zu lindern, gilt es, bestimmte Regeln im Umgang mit adipösen Patienten zu beachten. Von großer Bedeutung ist es, eine Atmosphäre zu schaffen, in der sich der Patient wohlfühlt. Hierzu zählen zunächst grundlegende Dinge, wie eine medizinische Ausstattung, die für adipöse Patienten geeignet ist. Dies beginnt beispielsweise mit stabilen, nicht durch zu enge Lehnen begrenzten Stühlen, mit tragfähigen, breiten Untersuchungsliegen sowie Patientenkitteln in Übergröße. Messinstrumente wie Maßbänder und Waagen sollten den Messbereich auch der extremen Adipositas abdecken.

\section{Die medizinische Ausstattung sollte an die Bedürf- nisse von adipösen Patienten angepasst sein.}

Neben diesen Ausstattungserfordernissen ist es wichtig, dass die Übergewichtsthematik mit Sensibilität behandelt wird. Viele Patienten leiden unter Schamund Versagensgefühlen hinsichtlich ihres Gewichts und ihrer Figur. Um diese Gefühle nicht weiter zu verstärken, sollte eine nicht-stigmatisierende Grundhaltung eingenommen werden. Dabei ist es hilfreich, sich vor Augen zu führen, dass adipöse Menschen nicht selbstverschuldet übergewichtig sind, sondern dass die Adipositas aus einem komplexen Zusammenspiel von genetischen, Umwelt- und Verhaltensbedingungen entsteht.

Für einen sensiblen Umgang mit adipösen Patienten empfiehlt es sich, die eigenen Einstellungen gegenüber Übergewichtigen zu reflektieren. Stellen Sie sich zum Beispiel die folgenden Fragen (adaptiert nach Puhl u. Brownell [0.J.]):

- Behandle ich einen Patienten oder sehe ich vor allem einen „Dicken“? 
- Würde ich diesen Patienten genauso behandeln, wenn er $20 \mathrm{~kg}$ weniger wiegen würde?

- Ziehe ich negative Rückschlüsse vom Gewicht auf Intelligenz, Compliance, Gesundheit, Lebensstil oder Charaktereigenschaften?

- Wie erkläre ich mir die Entstehung der Adipositas bei diesem Patienten?

- Fühle ich mich bei der Behandlung übergewichtiger Patienten weniger wohl als bei der Behandlung normalgewichtiger Patienten?

- Bin ich mir der besonderen Bedürfnisse und Sorgen adipöser Patienten bewusst?

Um auch dem Patienten eine nicht-wertende Grundhaltung gegenüber seinem eigenen Übergewicht vermitteln zu können, sollte bei Untersuchungen, z.B. beim Wiegen oder Messen des Bauchumfangs, sensibel vorgegangen werden. Sprachliche Abwertungen von Adipositas gilt es grundsätzlich zu vermeiden, wozu z.B. Wörter wie „Fettsein“ oder „Gewichtsproblem" zählen. Das Gewicht kann beispielsweise ohne Kommentar notiert werden.

\section{Das Wiegen}

Zunächst ist sicherzustellen, dass die Waage den Messbereich auch der extremen Adipositas abdeckt. Schamgefühle des Patienten können verhindert werden, indem die Waage an einem Ort aufgestellt ist, der Privatheit ermöglicht. Die zuständige Person sollte den Grund für das Wiegen erklären und nach dem Einverständnis des Patienten fragen. Während des Vorgangs ist eine sensible Kommunikation zielführend. Sprachinhalte, Mimik, Gestik und Tonfall sollten freundliche Zugewandtheit, keinesfalls aber Schock oder Ekel ausdrücken.

Wie in Kapitel III.2.1 beschrieben, sprechen nur wenige Ärzte das Übergewicht des Patienten überhaupt an. In der Tat fühlen sich Patienten bei diesem Thema leicht angegriffen, sodass dieser Problembereich Feingefühl erfordert. Eine Konfrontation eines Patienten mit der Thematik kann eine Abwehrhaltung auslösen. Das meist schon existierende Gefühl der Hilflosigkeit in Bezug auf das außer Kontrolle geratene Gewicht sollte nicht noch weiter gesteigert werden, sondern stattdessen sollte eine Motivation für Verhaltensänderungen geschaffen werden. Dies kann der Behandler durch offene Fragen erreichen, indem er nach dem Wohlbefinden des Patienten fragt, seine Erlaubnis einholt, das Thema Gewicht anzusprechen, es im Zusammenhang mit der Gesundheit thematisiert und die Änderungsbereitschaft des Patienten eruiert und verstärkt, wie in Tabelle 1 dargestellt (1. bis 5. Schritt). Es ist nicht davon auszu- gehen, dass eine Änderungsmotivation bereits besteht, sondern oft muss sie erst durch das Erfragen von Zielen und Problemen geweckt werden.

Der simple Ratschlag abzunehmen, ohne auf die Komplexität des Problems einzugehen, ist meist kontraproduktiv. Effektiver ist es, Informationen über die Entstehung von Adipositas (z.B. multifaktorielle Ursachen) und allgemeine Schwierigkeiten der Gewichtsregulation zu geben (z.B. Verfügbarkeit von Nahrung, Zeitmangel, Stress). Auch Schwierigkeiten bei der Gewichtsreduktion und beim Halten des reduzierten Gewichts sollten thematisiert werden, denn die Adipositas ist in der Tat eine langfristig schwer behandelbare Gesundheitsstörung.

Dennoch ist es angebracht, das Übergewicht und die damit verbundenen gesundheitlichen Risiken zur Sprache zu bringen. Dem Patienten kann dafür die eigene Besorgnis um seine Gesundheit mitgeteilt werden (6. Schritt). Damit die Informationsvermittlung nicht wie eine Belehrung wirkt, ist es hilfreich, offene Fragen zu stellen (z.B. „Wie denken Sie über das Risiko, an Diabetes zu erkranken?“). Auf diese Weise kann der Patient selbst eine Änderungsmotivation entwickeln.

Der bloße Hinweis, dass eine Gewichtsreduktion angeraten ist, stößt bei Patienten häufig auf Widerstand, da viele Patienten bereits zahlreiche, langfristig wenig erfolgreiche Diätversuche hinter sich haben. Anzuraten ist daher eher, gemeinsam zu eruieren, ob und wie der Patient sein Ernährungs- und Bewegungsverhalten so verändern kann, dass er sich wohlfühlt und zugleich sein Gewicht langfristig stabilisieren bzw. ein wenig senken kann $(5-10 \%$ des Ausgangsgewichts; Deutsche Adipositas-Gesellschaft 2007; 7. Schritt). Indem beispielsweise regelmäßig zu bestimmten Tageszeiten gegessen wird, kann ein zu großes Hungergefühl vermieden werden, was die Gefahr des Überessens reduziert. Weil viele Menschen unrealistische Idealvorstellungen bezüglich ,gesunder“ Ernährung haben, ist zu betonen, dass es nicht um das Umsetzen einer mutmaßlich idealen Ernährungsweise geht, auch nicht um das Erreichen des gesellschaftlichen Schlankheitsideals, sondern langfristig um persönliches Wohlfühlen und Gesundheit.

\section{Der Patient sollte für jegliche Verhaltensänderung in Richtung auf das Zielverhalten gelobt werden.}

Der Patient sollte für das Herstellen der Änderungsmotivation ausführlich zu seinen Zielen und Wünschen befragt werden (8. Schritt). Berichtet der Patient 
Tab. 1 Gesprächsführungsstrategien bei Adipositas (nach Hilbert et al. 2005)

Schritte Beispielfragen

1. Stellen Sie offene Fragen „Wie geht es Ihnen heute?“

2. Bitten Sie um Erlaubnis, das Thema Adipositas anzusprechen

„Wären Sie damit einverstanden, kurz über Ihr Gewicht zu sprechen und auch darüber wie es Ihrer Meinung nach Ihre Gesundheit beeinflusst?“

3. Bringen Sie die Änderungsbereitschaft des Patienten in Erfahrung

4. Verstärken Sie die Änderungsbereitschaft

5. Vermitteln Sie dem Patienten Informationen über Adipositas

6. Teilen Sie dem Patienten Ihre Bedenken mit

„Wie geht es Ihnen mit Ihrem Gewicht?“, „Wie bereit wären Sie, Ihre Essgewohnheiten zu verändern?“, „Wie bereit wären Sie, Ihre Bewegungsgewohnheiten zu verändern?“

„Ihr Gewicht liegt momentan im Bereich der Adipositas. Gibt es etwas, das Sie über Adipositas wissen möchten?", „Gibt es etwas, das Sie an Ihrem Gewicht mögen?", „Möchten Sie mir einige dieser Dinge erzählen?“, „Was mögen Sie nicht an Ihrem Gewicht?“, „Welche Befürchtungen haben Sie bezüglich Ihres Gewichts?“

„Adipositas erhöht das Risiko verschiedener medizinischer Probleme, wie z.B. Bluthochdruck, Schlaganfall, Krebs, Diabetes und Atemwegserkrankungen. Dies bedeutet nicht, dass Sie daran erkranken werden, sondern dass das Risiko für Sie größer ist, daran zu erkranken.“, „Wie denken Sie darüber?“

„Ich mache mir Sorgen, dass Ihre Ess- und Bewegungsgewohnheiten Ihre Gesundheit beeinträchtigen.“, „Ich mache mir Sorgen, dass Ihr Gewicht weiter steigt und Ihren Diabetes verschlechtert."

7. Unterstützen Sie die Veränderung des Gesundheitsverhaltens

„Hier sind einige Möglichkeiten, dies zu verändern: Eine Balance zwischen Nahrungsaufnahme und Bewegung zu finden, kann dabei helfen das Gewicht zu stabilisieren und Ihre Gesundheit zu verbessern.“, „Was glauben Sie, was für Sie am besten funktionieren würde?“, „Welche Verhaltensweisen in Bezug auf Essen und Bewegung würden Sie gerne ändern?“, „Für weitergehende Unterstützung würde ich Ihnen eine Adipositasverhaltenstherapie empfehlen, denn eine medikamentöse Behandlung oder ein operativer Eingriff sind bei Ihnen nicht angezeigt."

8. Motivieren Sie den Patienten, sein Gewicht zu stabilisieren oder zu

„Welches Ziel möchten Sie gerne erreichen?“, „Welche positiven Veränderungen erwarten Sie von einer Gewichtsreduktion?“, „Es wurde gezeigt, dass schon eine reduzieren Reduktion von 5-10\% Gesundheitsrisiken vermindert und die Gesundheit verbessert."

über Verhaltensänderungen, so sollte jegliche Änderung in Richtung auf eine bewusste Gewichtsregulation verstärkt werden:

Patient: „Seit letzter Woche habe ich damit begonnen, die Spaziergänge mit meinem Hund auszudehnen. Ich gehe nun jeden Tag eine halbe Stunde spazieren, statt nur eine Viertelstunde."

In dieser Situation ist es wichtig, dass der Arzt den Patienten lobt und hervorhebt, dass dies ein guter Schritt in Richtung Gesundheit ist. Der Patient kann ermutigt werden, weitere Schritte in dieser Richtung zu gehen und die Aktivität langsam und stabil zu steigern, um noch fitter zu werden. Der Arzt kann auch mit dem Patienten herausarbeiten, welche Bewegungsarten infrage kommen und wann, wo und wie lange der Patient sie umsetzen möchte, sodass er sich gut damit fühlt. Während das Loben für jegliche Art von Verhaltensänderung wesentlich ist, sollte das Bewegungsverhalten nicht als unzureichend zur Gewichtsreduktion, kritisiert werden.

Insgesamt erweist es sich als hilfreich, wenn nicht die Gewichtsreduktion an sich in den Vordergrund des Gesprächs gestellt wird, sondern jeweils auf das Verhalten fokussiert wird. Dem Patienten sollte vermittelt werden, dass er etwas an seinem Verhalten ändern kann (z.B. eine Portion Obst mehr am Tag anstelle von Süßem). In diesem Zusammenhang kann für eine optimale Unterstützung des Patienten auch auf professionelle Hilfsangebote zur Gewichtsreduktion oder-stabilisierung hingewiesen werden.

Selbstwirksamkeit und Optimismus sollten in Bezug auf das eigene Verhalten gefördert werden. 
Da es sich bei Übergewicht um ein sensibles Thema handelt, sollte der Behandler versuchen, schnell zu erkennen, wenn dem Patienten das Gespräch unangenehm wird und er nicht mehr in der Lage ist, das Gesagte anzunehmen. Um dies zu vermeiden, ist es hilfreich, den Patienten selbst zu Wort kommen zu lassen und keinen Druck auszuüben. Dabei sind offene Fragen hilfreich, damit der Patient Tempo und Inhalt selbst bestimmen kann.

Fängt der Patient zum Beispiel an, lange Pausen zu machen, auf dem Stuhl herumzurutschen, zu erröten oder den Blickkontakt zu vermeiden, kann es helfen, das Thema zurückhaltender anzusprechen.

\section{Zusammenfassend sollte es Ziel des Gesprächs sein, dass sich der Patient nicht stigmatisiert, sondern ernst genommen fühlt. Sein Verständnis des Prob- lems sollte erweitert, seine Ziele und Handlungsmög- lichkeiten transparenter, und seine Änderungsmoti- vation und Selbstwirksamkeit gesteigert werden.}

Wie mit anderen chronischen Krankheiten haben wir selten die Möglichkeit zu heilen, aber wir haben die Möglichkeit, den Patienten mit Respekt zu behandeln. Solch eine Erfahrung kann das größte Geschenk sein, das wir einem adipösen Patienten machen können (übersetzt nach Stunkard 1993).

\section{Literatur}

Befort CA, Greiner KA, Hall S, Pulvers KM, Nollen NL, Charbonneau A, Kaur H, Ahluwalia JS (2006) Weight-related perceptions among patients and physicians: How well do physicians judge patients' motivation to lose weight? | Gen Intern Med 21, 10861090

Brownell KD, Puhl R, Schwartz MB, Rudd L (2005) Weight bias: Nature, consequences, and remedies. Guilford Press New York

Crandall CS (1994) Prejudice against fat people: Ideology and selfinterest. J Pers Soc Psychol 66, 882-894
Deutsche Adipositas-Gesellschaft (2007) Prävention und Therapie der Adipositas. URL http://www.adipositas-gesellschaft.de/ fileadmin/PDF/Leitlinien/Adipositas-Leitlinie-2007.pdf (abgerufen am 07. Januar 2012)

Drury CA, Louis M (2002) Exploring the association between body weight, stigma of obesity, and health care avoidance. I Am Acad Nurse Pract 14, 554-561

Ferrante JM, Piasecki AK, Ohman-Strickland PA, Crabtree BF (2009) Family physicians' practices and attitudes regarding care of extremely obese patients. Obesity $17,1710-1716$

Hebl MR, Xu J (2001) Weighing the care: Physicians' reactions to the size of a patient. Int I Obes (Lond) 25, 1246-1252

Hebl MR, Xu J, Mason MF (2003) Weighing the care: Patients' perceptions of physician care as a function of gender and weight. Int | Obes 27, 269-275

Hilbert A (2008) Soziale und psychosoziale Auswirkungen der Adipositas: Stigmatisierung und soziale Diskriminierung. In: Herpertz S, de Zwaan M, Zipfel S (Hrsg.) Handbuch der Essstörungen und Adipositas 288-291. Springer Berlin

Hilbert A, Schnur A, Wilfey DE (2005) Obesity and binge eating disorder. In: Rubin EH, Zorumski CF (Hrsg.) Adult Psychiatry 247260. Blackwell Publishing Oxford

Hilbert A, Ried I (2009) Obesity in print: An analysis of German newspapers. Obes Facts 2, 46-51

Hilbert A, Rief W, Brähler E (2008) Stigmatizing attitudes towards obesity in a representative population-based sample: Prevalence and psychosocial determinants? Obesity 16, 1529-1534

Puhl RM, Brownell KD (2001) Bias, discrimination, and obesity. Obes Res 9, 788-805

Puhl RM, Brownell KD (2006) Confronting and coping with weight stigma: An investigation of overweight and obese adults. Obesity $14,1802-1815$

Puhl RM, Brownell KD (o.J.) Weight Bias in Health Care Settings. URL: http://www.yaleruddcenter.org/resources/bias_toolkit/ toolkit/Module-3/3-03a-Overview-Slides.pdf (abgerufen am 24. April 2011)

Puhl RM, Heuer CA (2009) The stigma of obesity: A review and update. Obesity 17, 941-964

Puhl RM, Latner JD (2007) Stigma, obesity, and the health of the nation's children. Psychol Bull 133, 557-580

Stunkard AJ (1993) Talking with patients. In: Stunkard AJ, Wadden TA (Hrsg.) Obesity: Theory and therapy (Bd. 2) 355-363. Raven Press New York

Wear D, Aultman JM, Varley JD, Zarconi I (2006) Making fun of patients: Medical students' perceptions and use of derogatory and cynical humor in clinical settings. Acad Med 81, 454-446 


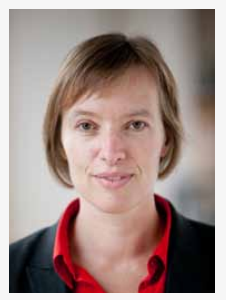

\section{Prof. Dr. rer. nat. Anja Hilbert}

Nach dem Studium der Psychologie in Marburg und Nancy (Frankreich) arbeitete Anja Hilbert 1996-2002 als wissenschaftliche Mitarbeiterin an den Universitäten Marburg und Siegen. 2000 Promotion. 2003-2004 Gastwissenschaftlerin an der Washington University School of Medicine in St. Louis (USA). 2004 Wissenschaftliche Assistentin für klinische Psychologie und Psychotherapie an der Universität Bielefeld. 2005-2009 Leiterin der Nachwuchsforschergruppe „Psychosoziale, ethische und rechtliche Konsequenzen genetischer Befunde bei Adipositas" an der Philipps-Universität Marburg. 2007 Habilitation. 2010-2011 Professorin für Klinische Psychologie am Departement für Psychologie der Universität Fribourg, Schweiz. Seit 2011 Professur für Verhaltensmedizin am Integrierten Forschungs- und Behandlungszentrum AdipositasErkrankungen der Universitätsmedizin Leipzig.

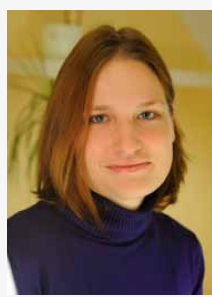

\section{M.Sc. Miriam Geiser}

Nach dem Bachelorstudium an der Universität Konstanz studierte sie von 2009 bis 2011 an der Université de Fribourg in der Schweiz und absolvierte dort ihren Masterabschluss in Klinischer und Gesundheitspsychologie. Währenddessen arbeitete sie als Hilfswissenschaftlerin am Lehrstuhl für Klinische Psychologie und Psychotherapie von Frau Prof. Dr. Anja Hilbert. Seit Oktober 2011 Weiterbildung und Vollzeittätigkeit in der Klinik am Korso (Fachzentrum für gestörtes Essverhalten) in Bad Oeynhausen. 